\title{
Bernoulli number identities from quantum field theory and topological string theory
}

\author{
Gerald V. Dunne and Christian Schubert
}

\begin{abstract}
We present a new method for the derivation of convolution identities for finite sums of products of Bernoulli numbers. Our approach is motivated by the role of these identities in quantum field theory and string theory. We first show that the Miki identity and the Faber-Pandharipande-Zagier (FPZ) identity are closely related, and give simple unified proofs which naturally yield a new Bernoulli number convolution identity. We then generalize each of these three identities into new families of convolution identities depending on a continuous parameter. We rederive a cubic generalization of Miki's identity due to Gessel and obtain a new similar identity generalizing the FPZ identity. The generalization of the method to the derivation of convolution identities of arbitrary order is outlined. We also describe an extension to identities which relate convolutions of Euler and Bernoulli numbers.
\end{abstract}

\section{Introduction: Convolution identities for Bernoulli numbers}

The Bernoulli numbers $B_{n}$ are defined by the generating function [1]

$$
b(x) \equiv \frac{x}{e^{x}-1}=\sum_{n=0}^{\infty} B_{n} \frac{x^{n}}{n !} .
$$

As is well known, the $B_{n}$ play an important role in combinatorics and number theory, and there exist many combinatorial identities involving these numbers $[2,3]$. In the present paper, we will be concerned with the special case of convolution identities, which involve finite sums of products of 
Bernoulli numbers. The best known such convolution identity was found already by Euler (and independently by Ramanujan):

Theorem 1.1 (Euler; Ramanujan). For integer $n \geq 2$,

$$
\sum_{k=1}^{n-1}\left(\begin{array}{l}
2 n \\
2 k
\end{array}\right) B_{2 k} B_{2 n-2 k}=-(2 n+1) B_{2 n}
$$

The proof follows directly from the definition (1.1), noting that the generating function $b(x)$ satisfies

$$
b(x)^{2}=(1-x) b(x)-x b^{\prime}(x)
$$

Equivalently, (1.2) follows by comparing the series expansions of either side of the trigonometric identity

$$
\operatorname{coth}^{2} x=1-(\operatorname{coth} x)^{\prime}
$$

Many more such identities, involving folded sums of Bernoulli numbers, have been found since Euler's work (see, e.g., [4-6]). Most of them are similar to Euler's identity (1.2) in the sense that they involve $\frac{B_{n}}{n !}$ rather than $B_{n}$ itself, as is the case already for the defining formula (1.1). Identities involving the $B_{n}$ themselves, without the factorial denominator, are much rarer. One such identity was found by Miki in 1978 [7].

Theorem 1.2 (Miki [7]). For integer $n \geq 2$,

$$
\sum_{k=1}^{n-1} \frac{B_{2 k} B_{2 n-2 k}}{(2 k)(2 n-2 k)}=\sum_{k=1}^{n-1} \frac{B_{2 k} B_{2 n-2 k}}{(2 k)(2 n-2 k)}\left(\begin{array}{c}
2 n \\
2 k
\end{array}\right)+\frac{B_{2 n}}{n} H_{2 n}
$$

Here $H_{i}$ denotes the $i$ th harmonic number,

$$
H_{i} \equiv \sum_{j=1}^{i} \frac{1}{j}
$$

As is well-known, the harmonic numbers can be alternatively expressed in terms of the digamma function $\psi(x)=\Gamma^{\prime}(x) / \Gamma(x)$ and the Euler-Mascheroni 
constant $\gamma$ :

$$
H_{i}=\psi(i+1)+\gamma
$$

Miki's original proof of the identity (1.5) identity is quite involved [7]. A more elementary proof was given recently by Gessel [8], using two different expressions for the Stirling numbers of the second kind. In Section 2.1, we present an even simpler proof, based on an appropriate generating function.

In 1998 Faber and Pandharipande [9] found that certain conjectural relations between Hodge integrals in Gromov-Witten theory [10,11] (see also [12]) require the following identity to hold.

Theorem 1.3 (Faber and Pandharipande, with a proof by Zagier [9]). For integer $n \geq 2$,

$$
\begin{aligned}
\sum_{k=1}^{n-1} \frac{\bar{B}_{2 k} \bar{B}_{2 n-2 k}}{(2 k)(2 n-2 k)} & =\frac{1}{n} \sum_{k=1}^{n} \frac{B_{2 k} \bar{B}_{2 n-2 k}}{(2 k)}\left(\begin{array}{l}
2 n \\
2 k
\end{array}\right)+\frac{\bar{B}_{2 n}}{n} H_{2 n-1} \\
\text { where } \quad \bar{B}_{n} & \equiv\left(\frac{1-2^{n-1}}{2^{n-1}}\right) B_{n} .
\end{aligned}
$$

A proof of the Faber-Pandharipande-Zagier (FPZ) identity (1.8) was given by Zagier in an appendix to [9]. Note that the structure of the FPZ identity (1.8) is similar to Miki's identity (1.5). We later show (see Theorem 2.1) that this similarity is even more striking if Miki's identity is written in a slightly different form.

Apart from pure mathematics, the Bernoulli numbers appear prominently in perturbative quantum field theory. This comes about at a very basic level [13]: perturbative loop calculations in quantum field theory generally involve traces of inverse powers of derivatives of functions defined on a circle. Since the spectrum of the ordinary derivative operator $\partial_{P}$ with periodic boundary conditions consists of the integer numbers, one has

$$
\operatorname{tr}\left(\partial_{P}^{-2 n}\right) \sim \sum_{k=1}^{\infty} \frac{1}{k^{2 n}}=\zeta(2 n)
$$

But $\zeta(2 n)$ is related to the Bernoulli numbers through Euler's identity,

$$
B_{2 n}=(-1)^{n+1} 2 \frac{(2 n) !}{(2 \pi)^{2 n}} \zeta(2 n)
$$


The Bernoulli numbers also appear naturally in so-called "effective action" computations in quantum field theory, a field pioneered by Heisenberg and Euler and Weisskopf [14].

In $[15,16]$, the present authors found that Miki's identity arises naturally in a certain computation in perturbative quantum field theory. Specifically, it arises in the course of the calculation of the two-loop effective Lagrangian for quantum electrodynamics in a constant background self-dual field. This calculation was done using two different integral representations. It turned out that both representations yield a result for the coefficients of the weak field expansion of this effective Lagrangian which involve a convolution of Bernoulli numbers, and that what is needed to show the equivalence of both results is precisely Miki's identity (1.5). Since the two integral representations used are related by a simple coordinate transformation, this actually yields a new, and quite straightforward, proof of Miki's identity. This proof will be given in Section 2.1. The simplicity of the approach presented here suggests a number of generalizations of these two identities, some of which are presented in Sections 3 to 5. It is straightforward to verify these identities explicitly using symbolic computer programs, and we have performed such checks for these identities for various values of $n$. Further generalizations are outlined in the conclusions.

\section{Simple generating function proofs of Miki and FPZ identities}

In this section we present simple unified proofs of the Miki and FPZ identities based on generating functions.

\subsection{Generating function proof of Miki's identity}

Consider the generating function

$$
\tilde{\psi}(x) \equiv \psi(x)-\ln x+\frac{1}{2 x} .
$$

This function plays an important role in the quantum field theory computations in $[15,16]$. From the asymptotic (large $x$ ) expansion of the digamma function [1] it follows that

$$
\tilde{\psi}(x) \sim-\sum_{k=1}^{\infty} \frac{B_{2 k}}{2 k} \frac{1}{x^{2 k}}
$$


Thus for the square of $\tilde{\psi}$, one finds

$$
[\tilde{\psi}(x)]^{2} \sim \sum_{n=2}^{\infty} \frac{1}{x^{2 n}} \sum_{k=1}^{n-1} \frac{B_{2 k} B_{2 n-2 k}}{(2 k)(2 n-2 k)} .
$$

Thus, we see that the $[\tilde{\psi}(x)]^{2}$ is the generating function for the left-hand side of Miki's identity (1.5). We prove Miki's identity by comparing (2.3) with the square of the following integral representation [see Equation 1.7.2 (25) in [17]] of $\tilde{\psi}(x)$ :

$$
\tilde{\psi}(x)=-\int_{0}^{\infty} d s \mathrm{e}^{-2 x s}\left(\operatorname{coth} s-\frac{1}{s}\right) .
$$

We break this comparison into three straightforward lemmas.

\section{Lemma 2.1.1.}

$$
\begin{aligned}
{[\tilde{\psi}(x)]^{2}=} & \int_{0}^{\infty} d y y e^{-2 x y} \int_{0}^{1} d u\left\{-1+2\left(\operatorname{coth} y-\frac{1}{y}\right)\left(\operatorname{coth} y u-\frac{1}{y u}\right)\right. \\
& \left.-\frac{2}{y(1-u)}\left[u\left(\operatorname{coth} y u-\frac{1}{y u}\right)-\left(\operatorname{coth} y-\frac{1}{y}\right)\right]\right\} .
\end{aligned}
$$

Proof. Squaring (2.4) we find

$$
\begin{aligned}
{[\tilde{\psi}(x)]^{2}=} & \int_{0}^{\infty} d s \int_{0}^{\infty} d s^{\prime} \mathrm{e}^{-2 x\left(s+s^{\prime}\right)} \\
& \times\left\{\operatorname{coth} s \operatorname{coth} s^{\prime}-\left(\frac{1}{s} \operatorname{coth} s^{\prime}+\frac{1}{s^{\prime}} \operatorname{coth} s\right)+\frac{1}{s s^{\prime}}\right\} .
\end{aligned}
$$

Now, using the trigonometric identity

$$
\operatorname{coth} s \operatorname{coth} s^{\prime}=\operatorname{coth}\left(s+s^{\prime}\right)\left(\operatorname{coth} s+\operatorname{coth} s^{\prime}\right)-1
$$

together with the symmetry $s \leftrightarrow s^{\prime}$, and the transformation of variables ${ }^{1}$

$$
y=s+s^{\prime}, \quad u=\frac{s^{\prime}}{s+s^{\prime}},
$$

\footnotetext{
${ }^{1}$ In quantum field theory terms, this change of variables corresponds to a change from a Feynman parameter integral (see, e.g., [18]) to a worldline parameter integral [19].
} 
it is straightforward to show that $[\tilde{\psi}(x)]^{2}$ can be rewritten as in Lemma 2.1.1. Note that the change of variables (2.8) introduces a Jacobian factor of $y$.

Our proof of Miki's identity follows by evaluating the asymptotic expansions of the integrals appearing on the RHS of (2.5).

\section{Lemma 2.1.2.}

$$
\begin{gathered}
2 \int_{0}^{\infty} d y y \mathrm{e}^{-2 x y} \int_{0}^{1} d u\left(\operatorname{coth} y-\frac{1}{y}\right)\left(\operatorname{coth} y u-\frac{1}{y u}\right) \\
\sim \sum_{n=2}^{\infty} \frac{1}{x^{2 n}} \sum_{k=1}^{n-1} \frac{B_{2 k} B_{2 n-2 k}}{(2 k)(2 n-2 k)}\left(\begin{array}{l}
2 n \\
2 k
\end{array}\right) .
\end{gathered}
$$

Proof. The $u$ integral is elementary:

$$
\int_{0}^{1} d u\left(\operatorname{coth} y u-\frac{1}{y u}\right)=\frac{1}{y} \ln \left(\frac{\sinh y}{y}\right) .
$$

After an integration by parts, the remaining $y$ integral takes the form

$$
2 x \int_{0}^{\infty} d y \mathrm{e}^{-2 x y} \ln ^{2}\left(\frac{\sinh y}{y}\right) .
$$

The asymptotic expansion of the $y$ integral is obtained using the Taylor expansion [1]

$$
\ln \left(\frac{\sinh y}{y}\right)=\sum_{k=1}^{\infty} \frac{2^{2 k-1} B_{2 k}}{k(2 k) !} y^{2 k}
$$

which directly yields the result (2.9) after performing the $y$ integration.

\section{Lemma 2.1.3.}

$$
\begin{aligned}
& -2 \int_{0}^{\infty} d y y \mathrm{e}^{-2 x y} \int_{0}^{1} d u\left\{\frac{1}{y(1-u)}\left[u\left(\operatorname{coth} y u-\frac{1}{y u}\right)-\left(\operatorname{coth} y-\frac{1}{y}\right)\right]\right\} \\
& \sim \sum_{n=1}^{\infty} \frac{1}{x^{2 n}} \frac{B_{2 n}}{n} H_{2 n} .
\end{aligned}
$$


Proof. First, consider the $u$ integral. We use the Taylor expansion of the coth function [1],

$$
y \operatorname{coth} y=\sum_{k=0}^{\infty} \frac{2^{2 k} B_{2 k}}{(2 k) !} y^{2 k}
$$

for both coth $y u$ and $\operatorname{coth} y$. The $u$ integral becomes elementary:

$$
\begin{array}{rl}
\int_{0}^{1} & d u \frac{\left[u\left(\operatorname{coth} y u-\frac{1}{y u}\right)-\left(\operatorname{coth} y-\frac{1}{y}\right)\right]}{(1-u)} \\
& =\sum_{n=1}^{\infty} \frac{B_{2 n} 2^{2 n} y^{2 n-1}}{(2 n) !} \int_{0}^{1} d u\left(\frac{u^{2 n}-1}{1-u}\right) \\
& =-\sum_{n=1}^{\infty} \frac{B_{2 n} 2^{2 n} y^{2 n-1}}{(2 n) !} H_{2 n} .
\end{array}
$$

Doing the $y$ integral we obtain the result of Lemma 2.1.3.

Miki's identity (1.5) is then proved by comparing the results of Lemmas 2.1.1 to 2.1.3 with (2.3).

We conclude this section on Miki's identity by remarking that in the proof of Lemma 2.1.2, the partial integration in $y$ leading to (2.11) is not essential. If, instead, one does the $y$ integral directly using (2.14) and (2.12), one arrives at a slightly different version of Miki's identity:

Theorem 2.1 (Modified form of Miki's identity). For integer $n \geq 2$,

$$
\begin{aligned}
\sum_{k=1}^{n-1} \frac{B_{2 k} B_{2 n-2 k}}{(2 k)(2 n-2 k)} & =\frac{1}{n} \sum_{k=1}^{n-1} \frac{B_{2 k} B_{2 n-2 k}}{(2 k)}\left(\begin{array}{c}
2 n \\
2 k
\end{array}\right)+\frac{B_{2 n}}{n} H_{2 n} \\
& =\frac{1}{n} \sum_{k=1}^{n} \frac{B_{2 k} B_{2 n-2 k}}{(2 k)}\left(\begin{array}{c}
2 n \\
2 k
\end{array}\right)+\frac{B_{2 n}}{n} H_{2 n-1}
\end{aligned}
$$

where we have used $H_{2 n}=H_{2 n-1}+\frac{1}{2 n}$, and $B_{0}=1$.

Comment 2.1.1. This last form (2.16) of Miki's identity brings out most clearly the similarity to the FPZ identity (1.8). 


\subsection{Generating function proof of the FPZ identity}

To prove the FPZ identity we use, instead of $\tilde{\psi}(x)$, the generating function $\bar{\psi}(x)$ defined by

$$
\bar{\psi}(x) \equiv \psi\left(x+\frac{1}{2}\right)-\ln x .
$$

The large $x$ expansion of $\bar{\psi}(x)$ is

$$
\bar{\psi}(x) \sim-\sum_{k=1}^{\infty} \frac{\bar{B}_{2 k}}{2 k} \frac{1}{x^{2 k}}
$$

where $\bar{B}_{2 k}$ was defined in (1.9). The expansion (2.18) follows from the corresponding expansion $(2.2)$ for $\tilde{\psi}(x)$, using the "doubling" identity [1] for the $\psi$ function,

$$
\psi(2 x)=\frac{1}{2} \psi(x)+\frac{1}{2} \psi\left(x+\frac{1}{2}\right)+\ln 2 .
$$

Thus, the square of $\bar{\psi}(x)$ is the generating function for the left-hand side of the FPZ identity (1.8):

$$
[\bar{\psi}(x)]^{2} \sim \sum_{n=2}^{\infty} \frac{1}{x^{2 n}} \sum_{k=1}^{n-1} \frac{\bar{B}_{2 k} \bar{B}_{2 n-2 k}}{(2 k)(2 n-2 k)}
$$

The generating function for the RHS of the FPZ identity is obtained by squaring the following integral representation for $\bar{\psi}(x)$ :

$$
\bar{\psi}(x)=-\int_{0}^{\infty} d s \mathrm{e}^{-2 x s}\left(\frac{1}{\sinh s}-\frac{1}{s}\right) .
$$

Lemma 2.2.1.

$$
\begin{aligned}
{[\bar{\psi}(x)]^{2}=} & 2 \int_{0}^{\infty} d y y \mathrm{e}^{-2 x y} \int_{0}^{1} d u\left\{\frac{1}{\sinh y}\left(\operatorname{coth} y u-\frac{1}{y u}\right)\right. \\
& \left.-\frac{1}{y(1-u)}\left[u\left(\frac{1}{\sinh y u}-\frac{1}{y u}\right)-\left(\frac{1}{\sinh y}-\frac{1}{y}\right)\right]\right\} .
\end{aligned}
$$


Proof. Squaring the integral representation (2.21), using the trigonometric identity

$$
\frac{1}{\sinh (s) \sinh \left(s^{\prime}\right)}=\frac{\operatorname{coth}(s)+\operatorname{coth}\left(s^{\prime}\right)}{\sinh \left(s+s^{\prime}\right)},
$$

and the symmetry under $s \leftrightarrow s^{\prime}$, it follows that

$$
\begin{aligned}
{[\bar{\psi}(x)]^{2}=} & 2 \int_{0}^{\infty} d s \int_{0}^{\infty} d s^{\prime} \mathrm{e}^{-2\left(s+s^{\prime}\right) x}\left\{\frac{1}{\sinh \left(s+s^{\prime}\right)}\left(\operatorname{coth}\left(s^{\prime}\right)-\frac{1}{s^{\prime}}\right)\right. \\
& \left.+\frac{1}{s}\left(\frac{1}{\sinh \left(s+s^{\prime}\right)}-\frac{1}{s+s^{\prime}}-\frac{1}{\sinh \left(s^{\prime}\right)}+\frac{1}{s^{\prime}}\right)\right\} .
\end{aligned}
$$

Applying the transformation of variables (2.8), we obtain (2.22).

Our proof of the FPZ identity now follows by evaluating the asymptotic expansions of the integrals appearing on the RHS of (2.22).

\section{Lemma 2.2.2.}

$$
\begin{gathered}
2 \int_{0}^{\infty} d y y \mathrm{e}^{-2 x y} \int_{0}^{1} d u\left\{\frac{1}{\sinh y}\left(\operatorname{coth} y u-\frac{1}{y u}\right)\right\} \\
\sim \sum_{n=1}^{\infty} \frac{1}{x^{2 n}} \frac{1}{n} \sum_{k=1}^{n} \frac{B_{2 k} \bar{B}_{2 n-2 k}}{(2 k)}\left(\begin{array}{c}
2 n \\
2 k
\end{array}\right) .
\end{gathered}
$$

Proof. The proof is almost identical to the proof of Lemma 2.1.2, but in doing the $y$ integral we use an asymptotic expansion of $1 / \sinh y$ rather than $\operatorname{coth} y$. This has the effect of replacing one of the Bernoulli number factors $B_{2 n-2 k}$ by $\bar{B}_{2 n-2 k}$, and also of changing the upper limit of the $k$ summation from $(n-1)$ to $n$.

\section{Lemma 2.2.3.}

$$
\begin{aligned}
& -2 \int_{0}^{\infty} d y y \mathrm{e}^{-2 x y} \int_{0}^{1} d u\left\{\frac{1}{y(1-u)}\left[u\left(\frac{1}{\sinh y u}-\frac{1}{y u}\right)-\left(\frac{1}{\sinh y}-\frac{1}{y}\right)\right]\right\} \\
& \sim \sum_{n=1}^{\infty} \frac{1}{x^{2 n}} \frac{\bar{B}_{2 n}}{n} H_{2 n-1} .
\end{aligned}
$$

Proof. The proof is almost identical to the proof of Lemma 2.1.3, except we use the asymptotic expansion of $1 / \sinh y$ rather than $\left(\operatorname{coth} y-\frac{1}{y}\right)$, which has the effect of replacing $B_{2 n}$ by $\bar{B}_{2 n}$. 
The FPZ identity (1.8) is then proved by comparing the results of Lemmas 2.2.1 to 2.2 .3 with (2.20). Note that the $n=1$ terms on the RHSs of (2.25) and (2.26) cancel, permitting the comparison with (2.20).

\section{A new convolution identity}

The similarity between the proofs and forms of the Miki and FPZ identities immediately suggests a new identity, in which on the left-hand side the $B_{2 n}$ and $\bar{B}_{2 n}$ are mixed. As is clear from Section 2, such an identity could be derived using the generating function $\tilde{\psi}(x) \bar{\psi}(x)$, and comparing its summation and integral representations. However, there is another, even simpler, way to derive this mixed identity. Note that the two generating functions $\tilde{\psi}(x)$ and $\bar{\psi}(x)$ are related via the $\psi$ function doubling identity (2.19) as:

$$
\tilde{\psi}(x)+\bar{\psi}(x)=2 \tilde{\psi}(2 x)
$$

Thus, it follows that

$$
2 \tilde{\psi}(x) \bar{\psi}(x)=4[\tilde{\psi}(2 x)]^{2}-[\tilde{\psi}(x)]^{2}-[\bar{\psi}(x)]^{2}
$$

A new Bernoulli convolution identity emerges by using the asymptotic expansions (2.2) and (2.18) for the left-hand side of (3.2), and the asymptotic expansions of the squares of the integral representations (2.4) and (2.21) of $\tilde{\psi}(x)$ and $\bar{\psi}(x)$, respectively, on the RHS. All necessary results for the squares of the relevant integral representations are contained in Lemmas 2.1.1 to 2.1.3 and 2.2.1 to 2.2.3. To express the result in a symmetrical form, we use the modified form of Miki's identity in (2.16).

Theorem 3.1. For integer $n \geq 2$ :

(3.3)

$$
\sum_{k=1}^{n-1} \frac{B_{2 k} \bar{B}_{2 n-2 k}}{(2 k)(2 n-2 k)}=\frac{1}{n} \sum_{k=1}^{n} \frac{B_{2 k} B_{2 n-2 k}}{(2 k)}\left(\begin{array}{c}
2 n \\
2 k
\end{array}\right)\left(\frac{1-2^{2 k-1}}{2^{2 n-1}}\right)+\frac{1}{n} \frac{B_{2 n}}{2^{2 n}} H_{2 n-1} .
$$

Proof. The generating function for the left-hand side is given by half the left-hand side of (3.2). The RHS is obtained by using Miki's identity in the form (2.16) for the squares of $\tilde{\psi}$, and the FPZ identity (1.8) for the square of $\bar{\psi}$. Simple algebra then leads to the form in (3.3). 


\section{Three infinite families of convolution identities}

The use of the generating functions $\tilde{\psi}(x)$ and $\bar{\psi}(x)$ to prove the Miki and FPZ identities, as well as the new "crossed" identity (3.3) in Theorem 3.1, immediately leads to natural generalizations of each type of identity.

\subsection{Generalization of Miki's Identity}

To derive a generalization of Miki's identity, consider the $p^{\text {th }}$ derivative of the generating function $\tilde{\psi}(x)$. This has the large $x$ asymptotic expansion:

$$
\tilde{\psi}^{(p)}(x) \sim(-1)^{p+1} \sum_{n=1}^{\infty} \frac{B_{2 n} \Gamma(2 n+p)}{(2 n) \Gamma(2 n)} \frac{1}{x^{2 n+p}} .
$$

This function also has the following integral representation:

$$
\tilde{\psi}^{(p)}(x)=-(-2)^{p} \int_{0}^{\infty} d s \mathrm{e}^{-2 x s} s^{p}\left(\operatorname{coth} s-\frac{1}{s}\right) .
$$

We can use this integral representation to extend the definition of $\tilde{\psi}^{(p)}(x)$ to non-integer values of $p$; for this extrapolation the expansion (4.1) continues to hold, as can be seen by using (2.14) under the integral in (4.2). Thus, in the following let $p$ denote an arbitrary non-negative number. We can derive new identities, for any such $p$, by squaring these two representations of $\tilde{\psi}^{(p)}(x)$, and then comparing, just as was done (for $p=0$ ) to prove Miki's identity. The proof proceeds in a very similar manner.

Lemma 4.1.1.

$$
\left[\tilde{\psi}^{(p)}(x)\right]^{2} \sim \sum_{n=2}^{\infty} \frac{1}{x^{2 n+2 p}} \sum_{k=1}^{n-1} \frac{B_{2 k} B_{2 n-2 k}}{(2 k)(2 n-2 k)} \frac{\Gamma(2 k+p) \Gamma(2 n-2 k+p)}{\Gamma(2 k) \Gamma(2 n-2 k)}
$$

Proof. follows from (4.1).

Lemma 4.1.2.

$$
\begin{aligned}
{\left[\tilde{\psi}^{(p)}(x)\right]^{2}=} & 2^{2 p} \int_{0}^{\infty} d y y^{2 p+1} \mathrm{e}^{-2 x y} \int_{0}^{1} d u u^{p}(1-u)^{p} \\
& \times\left\{-1+2\left(\operatorname{coth} y-\frac{1}{y}\right)\left(\operatorname{coth} y u-\frac{1}{y u}\right)\right. \\
& \left.-\frac{2}{y(1-u)}\left[u\left(\operatorname{coth} y u-\frac{1}{y u}\right)-\left(\operatorname{coth} y-\frac{1}{y}\right)\right]\right\}
\end{aligned}
$$


Proof. Square the integral representation (4.2), change variables from $s$ and $s^{\prime}$ to $y$ and $u$, as in (2.8), and regroup terms as in the proof of Lemma 2.1.1. Note that the argument about symmetrizing with respect to $s$ and $s^{\prime}$ still holds because the extra factors in the integrand appear as $\left(s s^{\prime}\right)^{p}=y^{2 p} u^{p}$ $(1-u)^{p}$.

Now consider each of the three terms appearing on the RHS of (4.4).

\section{Lemma 4.1.3.}

$$
-2^{2 p} \int_{0}^{\infty} d y y^{2 p+1} \mathrm{e}^{-2 x y} \int_{0}^{1} d u u^{p}(1-u)^{p} \sim-\frac{\Gamma^{2}(p+1)}{4 x^{2 p+2}} .
$$

Proof. immediate.

\section{Lemma 4.1.4.}

$$
\begin{gathered}
2^{2 p+1} \int_{0}^{\infty} d y y^{2 p+1} \mathrm{e}^{-2 x y} \int_{0}^{1} d u u^{p}(1-u)^{p}\left(\operatorname{coth} y-\frac{1}{y}\right)\left(\operatorname{coth} y u-\frac{1}{y u}\right) \\
\sim 2 \Gamma(p+1) \sum_{n=2}^{\infty} \frac{1}{x^{2 n+2 p}} \sum_{k=1}^{n-1} \frac{B_{2 k} B_{2 n-2 k}}{(2 k) !(2 n-2 k) !} \frac{\Gamma(2 k+p) \Gamma(2 n+2 p)}{\Gamma(2 p+2 k+1)} .
\end{gathered}
$$

Proof. First, consider the $u$ integral:

$$
\begin{array}{rl}
\int_{0}^{1} & d u u^{p}(1-u)^{p}\left(\operatorname{coth} y u-\frac{1}{y u}\right) \\
= & \sum_{n=1}^{\infty} \frac{B_{2 n} 2^{2 n} y^{2 n-1}}{(2 n) !} \int_{0}^{1} d u u^{2 n+p-1}(1-u)^{p} \\
= & \sum_{n=1}^{\infty} \frac{B_{2 n} 2^{2 n} y^{2 n-1}}{(2 n) !} \frac{\Gamma(p+1) \Gamma(p+2 n)}{\Gamma(2 p+2 n+1)} .
\end{array}
$$

Now doing the $y$ integral we obtain:

$$
\begin{array}{r}
2^{2 p+1} \sum_{n=1}^{\infty} \frac{B_{2 n} 2^{2 n}}{(2 n) !} \frac{\Gamma(p+1) \Gamma(p+2 n)}{\Gamma(2 p+2 n+1)} \int_{0}^{\infty} d y y^{2 p+2 n} \mathrm{e}^{-2 x y}\left(\operatorname{coth} y-\frac{1}{y}\right) \\
\sim 2^{2 p+1} \sum_{n=1}^{\infty} \frac{B_{2 n} 2^{2 n}}{(2 n) !} \frac{\Gamma(p+1) \Gamma(p+2 n)}{\Gamma(2 p+2 n+1)} \sum_{k=1}^{\infty} \frac{B_{2 k} 2^{2 k}}{(2 k) !} \frac{\Gamma(2 p+2 n+2 k)}{(2 x)^{2 p+2 n+2 k}}
\end{array}
$$

from which Lemma 4.1.4 follows. 


\section{Lemma 4.1.5.}

$$
\begin{aligned}
& -2^{2 p+1} \int_{0}^{\infty} d y y^{2 p+1} \mathrm{e}^{-2 x y} \int_{0}^{1} d u u^{p}(1-u)^{p} \\
& \quad \times\left\{\frac{1}{y(1-u)}\left[u\left(\operatorname{coth} y u-\frac{1}{y u}\right)-\left(\operatorname{coth} y-\frac{1}{y}\right)\right]\right\} \\
& \sim 2 \sum_{n=2}^{\infty} \frac{1}{x^{2 n+2 p}} \frac{B_{2 n} \Gamma(2 n+2 p)}{(2 n) !} \sum_{k=1}^{2 n} \beta(p+k, p+1) .
\end{aligned}
$$

Proof. First, consider the $u$ integral:

$$
\begin{gathered}
\int_{0}^{1} d u u^{p}(1-u)^{p-1}\left[u\left(\operatorname{coth} y u-\frac{1}{y u}\right)-\left(\operatorname{coth} y-\frac{1}{y}\right)\right] \\
\sim \sum_{n=1}^{\infty} \frac{B_{2 n} 2^{2 n} y^{2 n-1}}{(2 n) !} \int_{0}^{1} d u u^{p}(1-u)^{p-1}\left(u^{2 n}-1\right) \\
\sim \sum_{n=1}^{\infty} \frac{B_{2 n} 2^{2 n} y^{2 n-1}}{(2 n) !} \sum_{k=1}^{2 n} \beta(p+k, p+1)
\end{gathered}
$$

where $\beta(p, q)$ is the Euler beta function. Doing the $y$ integral we obtain the result of Lemma 4.1.5.

We are now ready to state the generalization of Miki's identity:

Theorem 4.1. For any $p \geq 0$, and for integer $n \geq 2$ :

$$
\begin{aligned}
& \sum_{k=1}^{n-1} \frac{B_{2 k} B_{2 n-2 k}}{(2 k)(2 n-2 k)} \frac{\Gamma(2 k+p) \Gamma(2 n-2 k+p)}{\Gamma(2 k) \Gamma(2 n-2 k)} \\
& =2 \Gamma(p+1) \sum_{k=1}^{n} \frac{B_{2 k} B_{2 n-2 k}}{(2 k) !(2 n-2 k) !} \frac{\Gamma(2 k+p) \Gamma(2 n+2 p)}{\Gamma(2 p+2 k+1)} \\
& \quad+2 \frac{B_{2 n} \Gamma(2 n+2 p)}{(2 n) !} \sum_{k=1}^{2 n-1} \beta(p+k, p+1) .
\end{aligned}
$$

Proof. Follows by comparing the result of Lemma 4.1.1 with those of Lemmas 4.1.2 to 4.1.5.

Comment 4.1.1. When $p=0$ we recover from Theorem 4.1 Miki's identity in the form of Theorem 2.1 . 
Comment 4.1.2. When $p=1$ we obtain from Theorem 4.1 a convolution identity which just involves the Bernoulli numbers themselves on the left-hand side: for $n \geq 2$,

$$
\sum_{k=1}^{n} B_{2 k} B_{2 n-2 k}=\frac{1}{n+1} \sum_{k=1}^{n} B_{2 k} B_{2 n-2 k}\left(\begin{array}{l}
2 n+2 \\
2 k+2
\end{array}\right)+2 n B_{2 n} .
$$

\subsection{Generalization of the FPZ Identity}

To derive a generalization of the FPZ identity, consider the $p$ th derivative of the generating function $\bar{\psi}(x)$. This has the large $x$ asymptotic expansion:

$$
\bar{\psi}^{(p)}(x) \sim(-1)^{p+1} \sum_{n=1}^{\infty} \frac{\bar{B}_{2 n} \Gamma(2 n+p)}{(2 n) \Gamma(2 n)} \frac{1}{x^{2 n+p}} .
$$

This function also has the following integral representation:

$$
\bar{\psi}^{(p)}(x)=-(-2)^{p} \int_{0}^{\infty} d s \mathrm{e}^{-2 x s} s^{p}\left(\frac{1}{\sinh s}-\frac{1}{s}\right) .
$$

As in the Miki case, we can use (4.14) to define $\bar{\psi}^{(p)}(x)$ for non-integer $p$. We can then derive new identities, for any positive $p$, by squaring these two representations of $\bar{\psi}^{(p)}(x)$, and then comparing, just as was done (for $p=0$ ) to prove the FPZ identity. The proof proceeds in a very similar manner.

\section{Lemma 4.2.1.}

$$
\left[\bar{\psi}^{(p)}(x)\right]^{2} \sim \sum_{n=2}^{\infty} \frac{1}{x^{2 n+2 p}} \sum_{k=1}^{n-1} \frac{\bar{B}_{2 k} \bar{B}_{2 n-2 k}}{(2 k)(2 n-2 k)} \frac{\Gamma(2 k+p) \Gamma(2 n-2 k+p)}{\Gamma(2 k) \Gamma(2 n-2 k)} .
$$

Proof. follows from (4.13).

\section{Lemma 4.2.2.}

$$
\begin{aligned}
{\left[\bar{\psi}^{(p)}(x)\right]^{2}=} & 2^{2 p+1} \int_{0}^{\infty} d y y^{2 p+1} \mathrm{e}^{-2 x y} \int_{0}^{1} d u u^{p}(1-u)^{p} \\
& \times\left\{\frac{1}{\sinh y}\left(\operatorname{coth} y u-\frac{1}{y u}\right)-\frac{1}{y(1-u)}\right. \\
& \left.\times\left[u\left(\frac{1}{\sinh y u}-\frac{1}{y u}\right)-\left(\frac{1}{\sinh y}-\frac{1}{y}\right)\right]\right\}
\end{aligned}
$$


Proof. Square the integral representation (4.14), change variables from $s$ and $s^{\prime}$ to $y$ and $u$, and regroup terms as in the proof of Lemma 2.2.1.

Now consider each of the two terms appearing on the RHS of (4.16).

\section{Lemma 4.2.3.}

$$
\begin{gathered}
2^{2 p+1} \int_{0}^{\infty} d y y^{2 p+1} \mathrm{e}^{-2 x y} \int_{0}^{1} d u u^{p}(1-u)^{p}\left\{\frac{1}{\sinh y}\left(\operatorname{coth} y u-\frac{1}{y u}\right)\right\} \\
\sim 2 \Gamma(p+1) \sum_{n=1}^{\infty} \frac{1}{x^{2 n+2 p}} \sum_{k=1}^{n} \frac{B_{2 k} \bar{B}_{2 n-2 k}}{(2 k) !(2 n-2 k) !} \frac{\Gamma(2 k+p) \Gamma(2 n+2 p)}{\Gamma(2 p+2 k+1)}
\end{gathered}
$$

Proof. The proof is almost identical to the proof of Lemma 4.1.4, but in doing the $y$ integral we use an asymptotic expansion of $1 / \sinh y$ rather than $\operatorname{coth} y-\frac{1}{y}$. This has the effect of replacing one of the Bernoulli number factors $B_{2 n-2 k}$ by $\bar{B}_{2 n-2 k}$, and also of changing the upper limit of the $k$ summation from $(n-1)$ to $n$.

\section{Lemma 4.2.4.}

$$
\begin{aligned}
& -2^{2 p+1} \int_{0}^{\infty} d y y^{2 p+1} \mathrm{e}^{-2 x y} \int_{0}^{1} d u u^{p}(1-u)^{p} \\
& \quad \times\left\{\frac{1}{y(1-u)}\left[u\left(\frac{1}{\sinh y u}-\frac{1}{y u}\right)-\left(\frac{1}{\sinh y}-\frac{1}{y}\right)\right]\right\} \\
& \sim 2 \sum_{n=2}^{\infty} \frac{1}{x^{2 n+2 p}} \frac{\bar{B}_{2 n} \Gamma(2 n+2 p)}{(2 n) !} \sum_{k=1}^{2 n} \beta(p+k, p+1) .
\end{aligned}
$$

Proof. The proof is almost identical to the proof of Lemma 4.1.5, except we use the asymptotic expansion of $1 / \sinh y$ rather than $\operatorname{coth} y$.

We are now ready to state the generalization of the FPZ identity:

Theorem 4.2. For any $p \geq 0$, and for integer $n \geq 2$ :

$$
\begin{aligned}
& \sum_{k=1}^{n-1} \frac{\bar{B}_{2 k} \bar{B}_{2 n-2 k}}{(2 k)(2 n-2 k)} \frac{\Gamma(2 k+p) \Gamma(2 n-2 k+p)}{\Gamma(2 k) \Gamma(2 n-2 k)} \\
& \quad=2 \Gamma(p+1) \sum_{k=1}^{n} \frac{B_{2 k} \bar{B}_{2 n-2 k}}{(2 k) !(2 n-2 k) !} \frac{\Gamma(2 k+p) \Gamma(2 n+2 p)}{\Gamma(2 p+2 k+1)}
\end{aligned}
$$




$$
+2 \frac{\bar{B}_{2 n} \Gamma(2 n+2 p)}{(2 n) !} \sum_{k=1}^{2 n-1} \beta(p+k, p+1) .
$$

Proof. Follows by comparing the result of Lemma 4.2.1 with those of Lemmas 4.2 .2 to 4.2 .4 .

Comment 4.2.1. When $p=0$ we recover from Theorem 4.2 the FPZ identity (1.8).

Comment 4.2.2. When $p=1$ we obtain from Theorem 4.2 a convolution identity which just involves the Bernoulli numbers themselves on the lefthand side: for $n \geq 1$,

$$
\sum_{k=1}^{n} \bar{B}_{2 k} \bar{B}_{2 n-2 k}=\frac{1}{n+1} \sum_{k=1}^{n} B_{2 k} \bar{B}_{2 n-2 k}\left(\begin{array}{l}
2 n+2 \\
2 k+2
\end{array}\right)+2 n \bar{B}_{2 n}
$$

\subsection{Generalization of Theorem 3.1}

To generalize Theorem 3.1, we differentiate $p$ times the relation (3.1) connecting the two generating functions $\tilde{\psi}(x)$ and $\bar{\psi}(x)$. This leads to

$$
\tilde{\psi}^{(p)}(x)+\bar{\psi}^{(p)}(x)=2^{p+1} \tilde{\psi}^{(p)}(2 x) .
$$

This relation also holds true for non-integer positive $p$, as can be easily seen using the integral representations $(4.2),(4.14)$ for $\tilde{\psi}^{(p)}$ and $\bar{\psi}^{(p)}$ and the trigonometric identity

$$
\operatorname{coth}(s)+\frac{1}{\sinh (\mathrm{s})}=\operatorname{coth}\left(\frac{s}{2}\right)
$$

Squaring the relation (4.21) we obtain

\section{Lemma 4.3.1.}

$$
2 \tilde{\psi}^{(p)}(x) \bar{\psi}^{(p)}(x)=2^{2 p+2}\left[\tilde{\psi}^{(p)}(2 x)\right]^{2}-\left[\tilde{\psi}^{(p)}(x)\right]^{2}-\left[\bar{\psi}^{(p)}(x)\right]^{2}
$$

$(p \geq 0)$. This brings us to 
Theorem 4.3. For any $p \geq 0$, and for integer $n \geq 2$ :

$$
\begin{aligned}
& \sum_{k=1}^{n-1} \frac{B_{2 k} \bar{B}_{2 n-2 k}}{(2 k)(2 n-2 k)} \frac{\Gamma(2 k+p) \Gamma(2 n-2 k+p)}{\Gamma(2 k) \Gamma(2 n-2 k)} \\
& =2 \Gamma(p+1) \sum_{k=1}^{n} \frac{B_{2 k} B_{2 n-2 k}}{(2 k) !(2 n-2 k) !}\left(\frac{1-2^{2 k-1}}{2^{2 n-1}}\right) \frac{\Gamma(2 k+p) \Gamma(2 n+2 p)}{\Gamma(2 p+2 k+1)} \\
& \quad+\frac{B_{2 n} \Gamma(2 n+2 p)}{(2 n) ! 2^{2 n-1}} \sum_{k=1}^{2 n-1} \beta(p+k, p+1) .
\end{aligned}
$$

Proof. The proof follows by taking the product of the expansions of the two functions on the left-hand side of Lemma 4.3.1, and comparing with the expansions of the squares of the integral representations of the three terms appearing on the RHS of Lemma 4.3.1, using the results of Theorems 4.1 and 4.2 .

Comment 4.3.1. When $p=0$ we recover the identity in Theorem 3.1.

Comment 4.3.2. When $p=1$ we obtain from Theorem 4.3 a convolution identity which just involves the Bernoulli numbers themselves on the lefthand side: for $n \geq 1$,

$$
\begin{aligned}
\sum_{k=1}^{n-1} B_{2 k} \bar{B}_{2 n-2 k}= & \frac{1}{n+1} \sum_{k=1}^{n} B_{2 k} B_{2 n-2 k}\left(\frac{1-2^{2 k-1}}{2^{2 n-1}}\right)\left(\begin{array}{l}
2 n+2 \\
2 k+2
\end{array}\right) \\
& +(2 n-1) \frac{B_{2 n}}{2^{2 n}} .
\end{aligned}
$$

Comment 4.3.3. Note that in all the above the positiveness condition on $p$ was used only to avoid singularities. Theorems 4.1 to 4.3 actually hold true also for negative $p$ as long as none of the $\Gamma$-factors on either side becomes singular.

\section{Higher order convolution identities}

In the recent [8], Gessel shows the existence of an infinite tower of convolution identities involving multiple products of Bernoulli numbers, of which Miki's identity (1.5) is just the lowest order one. He also explicitly obtains the next element of this series, a triple product identity: 
Theorem 5.1 ([8], equation (4)). For integer $n \geq 3$,

$$
\begin{aligned}
\sum_{\substack{k+l+m=n \\
k, l, m \geq 1}} \frac{B_{2 k} B_{2 l} B_{2 m}}{(2 k)(2 l)(2 m)}= & \sum_{\substack{k+l+m=n \\
k, l, m \geq 1}} \frac{B_{2 k} B_{2 l} B_{2 m}}{(2 k)(2 l)(2 m)}\left(\begin{array}{c}
2 n \\
2 k, 2 l, 2 m
\end{array}\right) \\
& +3 H_{2 n} \sum_{k=1}^{n-1}\left(\begin{array}{c}
2 n \\
2 k
\end{array}\right) \frac{B_{2 k} B_{2 n-2 k}}{(2 k)(2 n-2 k)} \\
& +6 H_{2 n, 2} \frac{B_{2 n}}{2 n}-\left(n^{2}-\frac{3}{2} n+\frac{5}{4}\right) \frac{B_{2 n-2}}{(2 n-2)}
\end{aligned}
$$

Here

$$
H_{2 n, 2} \equiv \sum_{1 \leq i<j \leq 2 n} \frac{1}{i j} \quad\left(=\sum_{i=1}^{2 n-1} \frac{H_{l}}{l+1}\right)
$$

In our present approach, it is clear how to construct generalizations of the Miki and FPZ identities involving $N$-fold products of Bernoulli numbers:

(1) Take the $N$ th power of $\tilde{\psi}(x)$ (resp. $\bar{\psi}(x)$ ). The expansions (2.2) (resp. (2.18)) generate the $N-1$-fold convolution on the left-hand side of the identity,

$$
\sum_{n=N}^{\infty} \frac{1}{x^{2 n}} \sum_{\substack{\sum_{i=1}^{N} k_{i}=n \\ k_{1}, k_{2}, \ldots, k_{N} \geq 1}} \prod_{i=1}^{N} \frac{B_{2 k_{i}}}{2 k_{i}}
$$

(with $B_{l}$ replaced by $\bar{B}_{l}$ in the FPZ case).

(2) Use (2.4) (resp. (2.21)) to rewrite

$$
\begin{aligned}
(\tilde{\psi}(x))^{N}= & \int_{0}^{\infty} d s_{1} \int_{0}^{\infty} d s_{2} \cdots \int_{0}^{\infty} d s_{N} \mathrm{e}^{-2 x\left(s_{1}+s_{2}+\cdots+s_{N}\right)} \\
& \times \prod_{i=1}^{N}\left(\operatorname{coth}\left(\mathrm{s}_{\mathrm{i}}\right)-\frac{1}{\mathrm{~s}_{\mathrm{i}}}\right)
\end{aligned}
$$

(with coth replaced by $\frac{1}{\sinh }$ in the FPZ case).

(3) Use trigonometric identities together with the symmetry of the integrand under permutations of the variables $\left\{s_{1}, \ldots, s_{N}\right\}$ to rewrite the integrand in such a way that only the combinations $s_{1}+s_{2}+\cdots+s_{N}$, 
$s_{2}+s_{3}+\cdots+s_{N}, \ldots, s_{N-1}+s_{N}, s_{N}$ appear as arguments of trigonometric functions (it is easy to see that this is always possible).

(4) Perform the transformation of variables from $\left\{s_{1}, \ldots, s_{N}\right\}$ to $\left\{y, u_{1}\right.$, $\left.u_{2}, \ldots, u_{N-1}\right\}$ where

$$
\begin{aligned}
y & =s_{1}+s_{2}+\cdots+s_{N} \\
u_{M} & =\frac{s_{M+1}+s_{M+2}+\cdots+s_{N}}{s_{1}+s_{2}+\cdots+s_{N}}, \quad M=1, \ldots, N-1 .
\end{aligned}
$$

The Jacobi factor of this transformation is $y^{N-1}$.

(5) Use the Taylor expansions (2.14),(2.12) to do all integrals.

Let us carry this through explicitly for the case $N=3$. In the Miki case, after step 3 one finds

\section{Lemma 5.1.}

$$
\begin{aligned}
(\tilde{\psi}(x))^{3}= & \int_{0}^{\infty} d s_{1} \int_{0}^{\infty} d s_{2} \int_{0}^{\infty} d s_{3} \mathrm{e}^{-2 x\left(s_{1}+s_{2}+s_{3}\right)} \\
& \times\left\{6 C_{123} C_{23} C_{3}+\frac{6}{s_{1}}\left[C_{123}-\frac{s_{23}}{s_{123}} C_{23}\right] C_{3}\right. \\
& +\frac{6}{s_{2}} C_{123}\left[C_{23}-\frac{s_{3}}{s_{23}} C_{3}\right]+\frac{6}{s_{2}}\left[\frac{1}{s_{1}}\left(C_{123}-\frac{s_{23}}{s_{123}} C_{23}\right)\right. \\
& \left.\left.-\frac{1}{s_{12}}\left(C_{123}-\frac{s_{3}}{s_{123}} C_{3}\right)\right]-3 C_{3}-2 C_{123}-\frac{4}{s_{123}}\right\}
\end{aligned}
$$

Here for compactness we have introduced the abbreviations

$$
s_{23}=s_{2}+s_{3}, \quad s_{123}=s_{1}+s_{2}+s_{3}, \quad C_{(\cdot)}=\operatorname{coth}\left(s_{(\cdot)}\right)-\frac{1}{s_{(\cdot)}}
$$

Moreover, we have already combined terms in a way which facilitates the evaluation of the integrals (in particular, it avoids the appearance of spurious singularities).

After the transformation (5.5) the integrals can be done in a way which is completely analogous to the $N=2$ case treated in Section 2 . The result is a slight modified form of the identity (5.1), 
Theorem 5.2 (Modified form of Gessel's identity). For integer $n \geq 3$,

$$
\begin{aligned}
\sum_{\substack{k+l+m=n \\
k, l, m \geq 1}} \frac{B_{2 k} B_{2 l} B_{2 m}}{(2 k)(2 l)(2 m)}= & \frac{3}{2 n} \sum_{\substack{k+l+m=n \\
k, l, m \geq 1}} \frac{B_{2 k} B_{2 l} B_{2 m}}{(2 k)(2 l)}\left(\begin{array}{c}
2 n \\
2 k, 2 l, 2 m
\end{array}\right) \\
& +\frac{3}{n} H_{2 n} \sum_{k=1}^{n-1}\left(\begin{array}{c}
2 n \\
2 k
\end{array}\right) \frac{B_{2 k} B_{2 n-2 k}}{(2 k)} \\
& +6 H_{2 n, 2} \frac{B_{2 n}}{2 n}-\left(n^{2}-\frac{3}{2} n+\frac{5}{4}\right) \frac{B_{2 n-2}}{(2 n-2)} .
\end{aligned}
$$

Comment 5.1. The somewhat different form of the RHS compared to (5.1) is due to the same type of ambiguity (regarding integrations-by-parts) which was mentioned already at the end of Section 2.1.

The FPZ case is again similar, though slightly more complicated. After step three one finds that the integrand can be written in the following way,

\section{Lemma 5.2.}

$$
\begin{aligned}
(\bar{\psi}(x))^{3}= & \int_{0}^{\infty} d s_{1} \int_{0}^{\infty} d s_{2} \int_{0}^{\infty} d s_{3} \mathrm{e}^{-2 x\left(s_{1}+s_{2}+s_{3}\right)} \\
& \times\left\{6 S_{123} C_{23} C_{3}+\frac{6}{s_{1}}\left[S_{123}-\frac{s_{23}}{s_{123}} S_{23}\right] C_{3}+\frac{6}{s_{2}} S_{123}\left[C_{23}-\frac{s_{3}}{s_{23}} C_{3}\right]\right. \\
& +\frac{6}{s_{2}}\left[\frac{1}{s_{1}}\left(S_{123}-\frac{s_{23}}{s_{123}} S_{23}\right)-\frac{1}{s_{12}}\left(S_{123}-\frac{s_{3}}{s_{123}} S_{3}\right)\right]-2 S_{123}-\frac{4}{s_{123}} \\
& \left.+\frac{6}{s_{123}}\left[C_{23}-S_{23}\right] C_{3}+\frac{6}{s_{2} s_{123}}\left[C_{23}-S_{23}-C_{3}+S_{3}\right]\right\} .
\end{aligned}
$$

Here we have used one more abbreviation,

$$
S_{(\cdot)}=\frac{1}{\sinh \left(s_{(\cdot)}\right)}-\frac{1}{s_{(\cdot)}} .
$$

Theorem 5.3 (Cubic generalization of the FPZ identity). For integer $n \geq 3$,

$$
\begin{aligned}
\sum_{\substack{k+l+m=n \\
k, l, m \geq 1}} \frac{\bar{B}_{2 k} \bar{B}_{2 l} \bar{B}_{2 m}}{(2 k)(2 l)(2 m)}= & \frac{3}{2 n} \sum_{\substack{k+l+m=n \\
k, l, m \geq 1}} \frac{B_{2 k} B_{2 l} \bar{B}_{2 m}}{(2 k)(2 l)}\left(\begin{array}{c}
2 n \\
2 k, 2 l, 2 m
\end{array}\right) \\
& +\frac{3}{n} H_{2 n} \sum_{k=1}^{n-1}\left(\begin{array}{c}
2 n \\
2 k
\end{array}\right) \frac{B_{2 k} \bar{B}_{2 n-2 k}}{(2 k)}
\end{aligned}
$$




$$
\begin{aligned}
& +\frac{3}{2 n^{2}} \sum_{k=1}^{n-1}\left(\begin{array}{l}
2 n \\
2 k
\end{array}\right) \frac{B_{2 k}}{2 k}\left(B_{2 n-2 k}-\bar{B}_{2 n-2 k}\right) \\
& +\frac{3}{2 n^{2}} H_{2 n-1}\left(B_{2 n}-\bar{B}_{2 n}\right) \\
& +6 H_{2 n, 2} \frac{\bar{B}_{2 n}}{2 n}-\frac{2 n-1}{4} \bar{B}_{2 n-2} .
\end{aligned}
$$

Note again the similarity with the Miki case, (5.8).

Comment 5.2. It would be straightforward to extend both (5.8) and (5.11) to continuous families of identities along the lines of Section 4.

\section{Conclusions}

The method presented here allows one to derive, with relatively little effort, convolution identities for Bernoulli numbers of the quadratic type as well as higher order ones. Clearly we have not been able here to explore all its ramifications. For example, it should be possible to derive "mixed" identities such as (3.3) also at the cubic or higher level. Another possible direction is to use other generating functions to generate related identities involving the Euler numbers. The simplest such case comes from considering the generating function

$$
\begin{aligned}
g(x) & =\int_{0}^{\infty} d s \mathrm{e}^{-2 x s} \operatorname{sech} s \\
& \sim \sum_{n=0}^{\infty} \frac{E_{2 n}}{(2 x)^{2 n+1}} .
\end{aligned}
$$

Then it follows that

$$
[g(x)]^{2}=2 \int_{0}^{\infty} d y \mathrm{e}^{-2 x y} \frac{\ln \cosh y}{\sinh y}
$$

from which one finds

$\sum_{k=1}^{n} E_{2 k-2} E_{2 n-2 k}=\frac{2}{n} \sum_{k=1}^{n} \frac{B_{2 k} B_{2 n-2 k}}{k}\left(2^{2 k}-1\right) 2^{2 k-1}\left(1-2^{2 n-2 k-1}\right)\left(\begin{array}{l}2 n \\ 2 k\end{array}\right)$.

$(n \geq 1)$. Clearly one can generate many other such identities relating convolutions of Euler numbers to convolutions of Bernoulli numbers. 
We conclude by emphasizing again that the types of generating functions and identities discussed here show up naturally in perturbative quantum field theory computations at the second-order (or "two loop") level $[15,16,20]$. We expect related multiple convolution identities of higher order to play a similar role for higher-loop contributions to the effective Lagrangian in quantum electrodynamics beyond the two-loop level. The higher order FPZtype identities might correspond to new relations between Hodge integrals and thus be of relevance for topological quantum field theory and string theory.

Note added (January 2013): This paper was submitted to the arXiv in June 2004, as http://arxiv.org/abs/math/0406610 but was delayed in publication review. Since then some related work citing our preprint has appeared: In [21] a method based on generating functions, similar to the one introduced here, was used to derive a convolution identity for Bernoulli polynomials that generalizes both Miki's identity (1.5) and the FPZ identity (1.8). It was also outlined how to use the same method to generalize Theorem 4.1 to the (univariate) polynomial level. In [22] this convolution identity was further generalized to a bivariate one. In the same paper, it was noted that our (4.12) had already been stated as a conjecture by Matiyasevich in 1997 [23], and a bivariate polynomial generalization of this identity was obtained, too. In [24] Theorem 4.1 was further generalized to the bivariate polynomial level, and the bivariate quadratic convolution identity of [22] was generalized to a family of multivariate multiple convolution identities for Bernoulli polynomials at any order (at the cubic level those presumably also relate to the identities of Section 5, although no such claim was made in [24]). In [25] a modification of our generating function $\tilde{\psi}$ was pointed out that may lead to another type of generalization of Miki's identity. Finally, in [26] the known vanishing of a certain type of one-loop amplitudes in $N=4$ Super-Yang-Mills theory was used to derive yet another type of quadratic convolution identities involving Bernoulli numbers, not obviously related to any of the above. These results further strengthen the case for an ubiquitous role of Bernoulli number identities in perturbative quantum field theory.

\section{Acknowledgments}

We are very grateful to Richard Stanley for correspondence, and to Albrecht Klemm for discussions. C.S. thanks the Institut des Hautes Études Scientifiques, Bures-sur-Yvette, and the Albert-Einstein-Institut, Potsdam, for hospitality. G.D. thanks the US Department of Energy for support 
under grant DE-FG02-92ER40716, and thanks the Rockefeller Foundation for a Bellagio Residency Award. We also acknowledge the support of NSF and CONACyT for a US-Mexico collaborative research grant NSF-INT0122615 .

\section{References}

[1] M. Abramowitz and I. Stegun, Handbook of Mathematical Functions, Dover, New York, 1972.

[2] L. Comtet, Advanced Combinatorics, Dordrecht, Boston, 1974.

[3] T.M. Apostol, Introduction to Analytic Number Theory, Springer, New York, 1998.

[4] K. Dilcher, Sums of products of Bernoulli numbers, J. Number Theory 60 (1996), 23.

[5] I.-C. Huang and S.-Y. Huang, Bernoulli numbers and polynomials via residues, J. Number Theory 76 (1999), 178.

[6] A. Basu and T.M. Apostol, A new method for investigating Euler sums, Ramanujan J. 4 (2000), 397

[7] H. Miki, A relation between Bernoulli numbers, J. Number Theory 10 (1978), 297

[8] I.M. Gessel, On Miki's identity for Bernoulli numbers, J. Number Theory 110 (2005), 75

[9] C. Faber and R. Pandharipande, Hodge integrals and Gromov-Witten theory, Invent. Math. 139 (2000), 137; arXiv:math.AG/9810173.

[10] T. Eguchi, K. Hori and C.-S. Xiong, Quantum cohomology and Virasoro algebra, Phys. Lett. B 402 (1997), 71.

[11] E. Getzler and R. Pandharipande, Virasoro constraints and the Chern classes of the Hodge bundle, Nucl. Phys. B 530 (1998), 701; arXiv:math.AG/9805114.

[12] C. Liu, K. Liu and J. Zhou, Mariño-Vafa formula and Hodge integral identities, J. Alg. Geom. 15 (2006), 379; arXiv:math.AG/0308015.

[13] M.G. Schmidt and C. Schubert, On the calculation of effective actions by string methods, Phys. Lett. B 318 (1993), 438; arXiv: hep-th/9309055. 
[14] For a review, see : G.V. Dunne, Heisenberg-Euler effective Lagrangians: Basics and extensions, Ian Kogan Memorial Collection, From Fields to Strings: Circumnavigating Theoretical Physics, M.A. Shifman et al. (eds.) (2004), vol. I, 445, arXiv:hep-th/0406216.

[15] G.V. Dunne and C. Schubert, Two-loop self-dual Euler-Heisenberg Lagrangians (I): real part and helicity amplitudes, J. High Energy Phys. 08 (2002), 053; arXiv:hep-th/0205004.

[16] G.V. Dunne and C. Schubert, Two-loop self-dual Euler-Heisenberg Lagrangians (II): imaginary part and Borel analysis, J. High Energy Phys. 06 (2002), 042; arXiv:hep-th/0205005.

[17] A. Erdélyi (ed.), Higher transcendental functions, Vol. I, Kreiger, Florida, 1981.

[18] C. Itzykson and J. Zuber, Quantum field theory, McGraw-Hill, New York, 1985.

[19] M.G. Schmidt and C. Schubert, Worldline Green functions for multiloop diagrams, Phys. Lett. B 331 (1994), 69; arXiv:hep-th/9403158.

[20] G.V. Dunne, Two-loop diagrammatics in a self-dual background, J. High Energy Phys. 02 (2004), 013; arXiv:hep-th/0311167.

[21] M.C. Crabb, The Miki-Gessel Bernoulli number identity, Glasgow Math. J. 47 (2005), 327.

[22] H. Pan and Z.-W. Sun, New identities involving Bernoulli and Euler polynomials, J. Comb. Theory, A 113 (2006), 156.

[23] Y. Matiyasevich, Identities with Bernoulli numbers, http://logic.pdmi. ras.ru/ỹumat/Journal/Bernoulli/bernulli.htm, 1997.

[24] A.M. Fu, H. Pao and I.F. Zhang, Symmetric identities on Bernoulli polynomials, J. Number Theory 129 (2009), 2696; arXiv:0709.2593 [math.NT].

[25] H.G. Gadiyar and R. Padma, A comment on Matiyasevich's Identity \#0102 with Bernoulli numbers, arXiv:math.NT/0608675.

[26] A. Gorsky and A. Zhiboedov, Aspects of the $N=4 S Y M$ amplitude-Wilson polygon duality, Nucl. Phys. B 835 (2010), 343; arXiv:0911.3626 [hep-th]. 
Department of Physics and Department of Mathematics

University OF CONNECTICUT

Storrs, CT 06269

USA

E-mail address: gerald.dunne@uconn.edu

Institut des Hautes Études SCIEntifiques

LE BoIs-MARIE, 35,

F-91440 BuRES-SUR-YVETTE

FRANCE

Department of Physics and Geology

University of TeXAs PAN AMERICAN

EDINBURG,

TX 78541-2999, USA

Instituto DE Física y MATEMÁticas

Universidad Michoacana de San Nicolás de Hidalgo

Apdo. Postal 2-82

C.P. 58040, MORELIA

MichoACÁn, MÉXiCO

E-mail address: schubert@ifm.umich.mx

Received JAnuARY 22, 2013 
